\title{
CARACTERIZAÇÃO FENOLÓGICA E REQUERIMENTOS TÉRMICOS DE VARIEDA- DES DE UVAS SEM SEMENTES NO VALE DO SÃO FRANCISCO ${ }^{1}$
}

\author{
PATRICIA COELHO DE SOUZA LEÃO² \& EMANUEL ELDER GOMES DA SILVA³
}

\begin{abstract}
RESUMO - O comportamento fenológico e requerimentos térmicos de variedades de uvas sem sementes foram estudados nas condições do Vale do São Francisco durante os anos de 2001-2002, em seis diferentes datas de poda (12/2000, 04/2001, 06/2001, 10/2001, 01-02/2002 e 04/2002). O experimento foi realizado no Campo Experimental de Bebedouro, em Petrolina-PE. As variedades utilizadas foram Superior Seedless (Festival), Thompson Seedless, Catalunha, Perlette e Marroo Seedless, enxertadas sobre porta-enxerto IAC 572 ('Jales'). Avaliou-se a duração em dias dos estádios fenológicos, gemas dormentes (data de poda) a gemas inchadas (1), gemas inchadas a início de brotação (2), início de brotação a 5-6 folhas separadas (3), 5-6 folhas separadas a início de floração (4), início de floração à plena-floração (5), plena-floração a "chumbinho" (6), "chumbinho" à "ervilha" (7), "ervilha" a 1/2 baga (8), 1/2 baga a início de maturação (9) e início de maturação à maturação plena (10). Os requerimentos térmicos foram obtidos em termos de grausdia (GD) necessários para atingir cada fase fenológica a partir da poda. O ciclo fenológico médio variou de 89 dias e 1.315 GD na variedade Superior Seedless a 105 dias e 1.514 GD na variedade Perlette, destacando-se as variedades Superior Seedless e Marroo Seedless como precoces e as demais com ciclo intermediário. Os períodos compreendidos entre o início e final da maturação, $1 / 2$ baga a início de maturação e 5 a 6 folhas separadas a início de floração apresentaram a maior duração entre todos os subperíodos em todas as variedades.
\end{abstract}

Termos para indexação: fenologia, uvas sem sementes, requerimentos térmicos

\section{PHENOLOGICAL EVALUATION AND THERMAL REQUIREMENTS OF FIVE SEEDLESS GRAPES IN THE SAN FRANCISCO RIVER VALLEY}

\begin{abstract}
The phenological behaviour and heat summation requirements of seedless grapes were studied in the San Francisco River Valley during the seasons 2001-2002, for six pruning dates (12/2000,04/2001,06/2001, 10/2001, 01 and 02/2002 and 04/2002). The varieties evaluated were Superior Seedless (Festival), Thompson Seedless, Catalunha, Perlette and Marroo Seedless, grafted on IAC 572 (' Jales'). The number of days were evaluated for the following phenological phases: bud dorment to bud swelling (1), bud swelling to bud breaking (2), bud breaking to five to six leaves unfolded (3), five to six leaves unfolded to beginning of flowering (4), beginning of flowering to ending of flowering (5), ending of flowering to fruit set (pellet-like berries) (6), fruit set to the pea-size berries (7), pea-size berries to beginning of bunch touch (8), beginning of bunch touch to beginning of ripenning (9) and beginning of ripenning to berries ripe (10). Heat summation requirements were observed in degree-days (DG) necessary to start each phenological phase from the pruning date until the harvest. The average phenological cycle varied of 89 days and 1315 DG in the Superior Seedless to 105 days and 1514 DG in the Perlette. The periods among the beginning and ending of the maturation, beginning of bunch touch to beginning of maturation and 4 to 6 leaves separated to beginning of flowering presented the largest duration among all the subperíodos in all varieties. Index terms: phenology, seedless grape, heat requirements
\end{abstract}

\section{INTRODUÇÃO}

$\mathrm{Na}$ introdução de novas variedades, a fenologia desempenha importante função, pois permite a caracterização da duração das fases do desenvolvimento da videira em relação ao clima, especialmente às variações estacionais, além de ser utilizada para interpretar como as diferentes regiões climáticas interagem com a cultura (Terra et al., 1998).

$\mathrm{Na}$ viticultura brasileira realizaram-se estudos de fenologia e caracterização térmica nas tradicionais regiões produtoras de uva no sudeste e sul do país. Mandelli (1984) caracterizou a potencialidade climática da região de Bento Gonçalves no Rio Grande do Sul para o cultivo de algumas variedades para vinificação. A fenologia da variedade Niagara Rosada foi estudada por Pedro Júnior et al. (1993), Ferri (1994) e Guerreiro (1997). Por sua vez, Silva et al. (1990) estudaram o comportamento de vinte variedades americanas de videira em Jundiaí. Boliani (1994) avaliou o comportamento fenológico das variedades Itália e Rubi na região oeste do Estado de São Paulo. No Vale do São Francisco, seis cultivares de uvas sem sementes foram avaliadas em condições tropicais (Leão e Pereira, 2001).

A fenologia varia em função do genótipo e das condições climáticas de cada região produtora, ou em uma mesma região devido às variações estacionais do clima ao longo do ano. Em condições de clima tropical, como aquelas predominantes no Vale do São Francisco, a videira vegeta continuamente, não apresentando fase de repouso hibernal. A data de poda passa a ser a referência para o início do ciclo fenológico da videira, que sofre a influência das condições climáticas predominantes durante aquele período. No entanto, poucos estudos foram realizados sobre a fenologia da videira em condições tropicais.
A caracterização das exigências térmicas da videira mediante o conceito de graus-dia tem sido utilizada por diversos autores, sendo um método eficiente para prever antecipadamente a data de colheita (Pedro Júnior et al, 1994).

O presente trabalho teve como objetivo estudar o comportamento fenológico de cinco variedades de uvas sem sementes em diferentes épocas de poda nas condições do Vale do São Francisco.

\section{MATERIALE MÉTODOS}

O experimento foi conduzido no Campo Experimental de Bebedouro, pertencente à Embrapa Semi-Árido, localizado no município de Petrolina-PE (latitude $9^{\circ} 09^{\prime}$ Sul, longitude $40^{\circ} 22^{\prime}$ Oeste, e altitude média de $365 \mathrm{~m}$ ). Segundo Köeppen, o clima da região pode ser classificado como tipo Bswh, que corresponde a uma região semi-árida muito quente. As temperaturas mensais médias, mínimas e máximas que ocorreram durante a realização do trabalho são apresentadas na Figura 1.

O período considerado no estudo correspondeu aos anos de 2000 a 2002, estudando-se para cada variedade seis diferentes datas de poda: dezembro de 2000, abril de 2001, junho de 2001, outubro de 2001, janeiro a fevereiro de 2002 e abril de 2002.

Foram utilizadas as seguintes variedades de uva sem sementes: Superior Seedless ou Festival, Thompson Seedless, Catalunha, Perlette e Marroo Seedless, enxertadas sobre porta-enxerto IAC 572 'Jales'.

O comportamento fenológico das variedades foi avaliado através de observações visuais realizadas semanalmente a partir da poda até a colheita. As determinações dos estádios fenológicos foram baseadas

\footnotetext{
${ }^{1}$ (Trabalho 034/2003). Recebido: 07/02/2003. Aceito para publicação: 01/10/2003. Apoio financeiro FUNDECI-Banco do Nordeste.

${ }^{2}$ Eng ${ }^{a}$ Agr., M.Sc., Pesquisadora Embrapa Semi-Árido, Caixa Postal 23, 56302-970, Petrolina, PE, Fone: (87)3862-1711, patricia@cpatsa.embrapa.br.

${ }^{3}$ Enga Agr., M.Sc., Bolsista CNPq, Embrapa Semi-Árido, emanoel@cpatsa.embrapa.br.
} 


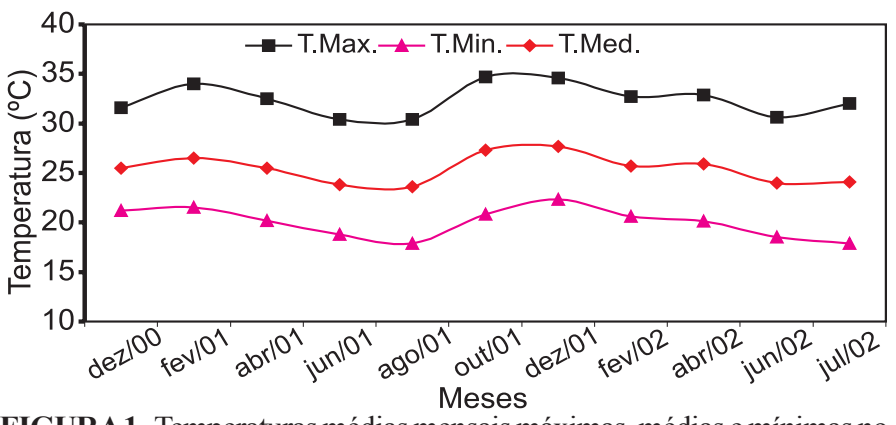

FIGURA1- Temperaturas médias mensais máximas, médias e mínimas no Campo Experimental de Bebedouro, Petrolina-PE (20002002)

na escala BBCH (Lorenz et al.,1995), para os seguintes períodos: gemas dormentes (data de poda) a gemas inchadas - fases 00 a 05 (1), gemas inchadas a início de brotação - fases 05 a 09 (2), início de brotação a 56 folhas separadas - fases 09 a 16 (3), 5-6 folhas separadas a início de floração - fases 16 a 61 (4), início de floração à plena-floração - fases 61 a 65 (5), plena-floração a "chumbinho"- fases 65 a 71 (6), "chumbinho" à "ervilha" - fases 71 a 75 (7), "ervilha" a $1 / 2$ baga - fases 75 a 79 (8), 1/2 baga a início de maturação - fases 79 a 81 (9) e início de maturação à maturação plena - fases 81 a 89 (10). O momento adequado para a colheita foi considerado quando as variedades apresentavam teor de sólidos solúveis totais superior a $16^{\circ}$ Brix.

Para caracterização dos requerimentos térmicos de cada variedade, utilizou-se o somatório de graus-dia desde a poda até a colheita para os seis ciclos de produção, bem como para cada um dos períodos, utilizando-se temperatura-base de $12^{\circ} \mathrm{C}$, segundo equações propostas por Villa Nova et al. (1972).

\section{RESULTADOS E DISCUSSÃO}

Observou-se uma variação no número de dias e requerimentos térmicos necessários para completar cada estádio fenológico, entre as diferentes variedades e épocas de poda (Tabelas 1 e 2). Essas variações eram esperadas uma vez que as variedades possuem origem genética distintas e foram obtidas para adaptação em condições ambientais diversas.

TABELA 1 - Número de dias para os períodos compreendidos entre as principais fases fenológicas de cinco variedades de uvas sem sementes, Petrolina-PE, 2000 a 2002.

\begin{tabular}{|c|c|c|c|c|c|c|c|c|c|c|c|c|c|}
\hline \multirow[t]{2}{*}{ Variedades } & \multirow{2}{*}{$\begin{array}{c}\text { Ano/ } \\
\text { Semestre }\end{array}$} & \multirow[b]{2}{*}{$\begin{array}{c}\text { Data } \\
\text { de poda }\end{array}$} & \multicolumn{11}{|c|}{ Fases Fenológicas } \\
\hline & & & 1 & 2 & 3 & 4 & 5 & 6 & 7 & 8 & 9 & 10 & Total \\
\hline & $2001 / 1^{\circ}$ & $18 / 12 / 2000$ & 7 & 3 & 3 & 18 & 3 & 5 & 9 & 16 & 9 & 13 & 86 \\
\hline & $2001 / 1^{\circ}$ & $25 / 04 / 2001$ & 12 & ---- & 3 & 12 & 6 & 5 & 5 & 12 & 10 & 25 & 90 \\
\hline Superior & $2001 / 2^{\circ}$ & $21 / 06 / 2001$ & 8 & 4 & 3 & 20 & 4 & 6 & 9 & 8 & 16 & 7 & 85 \\
\hline \multirow{7}{*}{ Seedless } & $2001 / 2^{\circ}$ & $29 / 10 / 2001$ & 5 & 2 & 5 & 17 & 4 & 3 & 7 & 10 & 14 & 18 & 85 \\
\hline & $2002 / 1^{\circ}$ & $29 / 01 / 2002$ & 5 & 4 & 5 & 14 & 4 & 6 & 5 & 11 & 15 & 31 & 100 \\
\hline & $2002 / 1^{\circ}$ & $09 / 04 / 2002$ & 6 & 3 & 4 & 20 & 2 & 3 & 6 & 8 & 24 & 21 & 97 \\
\hline & \multicolumn{2}{|c|}{ Média } & 7 & 3 & 4 & 17 & 4 & 5 & 7 & 11 & 15 & 19 & 91 \\
\hline & \multicolumn{2}{|c|}{ Desvio Padrão } & 3 & 1 & 1 & 3 & 1 & 1 & 2 & 3 & 5 & 7 & 9 \\
\hline & $2001 / 1^{\circ}$ & $19 / 12 / 2000$ & 6 & 3 & 3 & 18 & 4 & 5 & 10 & 14 & 11 & 37 & 111 \\
\hline & $2001 / 1^{\circ}$ & $18 / 04 / 2001$ & 9 & ---- & 4 & 17 & 3 & 7 & 4 & 9 & 19 & 28 & 100 \\
\hline \multirow{9}{*}{ Perlette } & $2001 / 2^{\circ}$ & $18 / 06 / 2001$ & 6 & 5 & 3 & 21 & 4 & 7 & 10 & 5 & 27 & 24 & 112 \\
\hline & $2001 / 2^{\circ}$ & $23 / 10 / 2001$ & 5 & 3 & 6 & 13 & 4 & 5 & 4 & 10 & 16 & 22 & 88 \\
\hline & $2002 / 1^{\circ}$ & $28 / 01 / 2002$ & 4 & 4 & 5 & 16 & 4 & 7 & 5 & 8 & 26 & 34 & 113 \\
\hline & $2002 / 1^{\circ}$ & $08 / 04 / 2002$ & 6 & 4 & 4 & 18 & 4 & 2 & 7 & 9 & 26 & 24 & 104 \\
\hline & \multicolumn{2}{|c|}{ Média } & 6 & 4 & 4 & 17 & 4 & 6 & 7 & 9 & 21 & 28 & 105 \\
\hline & \multicolumn{2}{|c|}{ Desvio padrão } & 2 & 1 & & 3 & $\mathbf{0}$ & 2 & 3 & 3 & 7 & 6 & 10 \\
\hline & $2001 / 1^{\circ}$ & $18 / 12 / 2000$ & 7 & 3 & 3 & 18 & 3 & 6 & 10 & 17 & 11 & 31 & 109 \\
\hline & $2001 / 1^{\mathrm{o}}$ & $19 / 04 / 2001$ & 8 & --- & 5 & 17 & 3 & 7 & 3 & 9 & 19 & 25 & 96 \\
\hline & $2001 / 2^{\circ}$ & $19 / 06 / 2001$ & 6 & 5 & 6 & 23 & 3 & 7 & 10 & 8 & 21 & 21 & 110 \\
\hline \multirow[t]{7}{*}{ Catalunha } & $2001 / 2^{\circ}$ & $24 / 10 / 2001$ & 5 & 3 & 6 & 15 & 5 & 3 & 5 & 16 & 16 & 23 & 97 \\
\hline & $2002 / 1^{\circ}$ & $31 / 01 / 2002$ & 7 & 4 & 4 & 18 & 5 & 3 & 5 & 8 & 21 & 23 & 98 \\
\hline & $2002 / 1^{\circ}$ & $10 / 04 / 2002$ & 5 & 3 & 4 & 19 & 3 & 4 & 6 & 13 & 20 & 24 & 101 \\
\hline & \multicolumn{2}{|c|}{ Média } & 6 & 4 & 5 & 18 & 4 & 5 & 7 & 12 & 18 & 25 & 102 \\
\hline & \multicolumn{2}{|c|}{ Desvio padrão } & 1 & 1 & 1 & 3 & 1 & 2 & 3 & 4 & 4 & 3 & 6 \\
\hline & $2001 / 1^{\circ}$ & $18 / 12 / 2000$ & 6 & 2 & 3 & 20 & 3 & 6 & 10 & 17 & 8 & 33 & 108 \\
\hline & $2001 / 1^{\circ}$ & $23 / 04 / 2001$ & 12 & ---- & 2 & 14 & 6 & 5 & 5 & 12 & 14 & 22 & 93 \\
\hline Thompson & $2001 / 2^{\circ}$ & $20 / 06 / 2001$ & 6 & 5 & 3 & 22 & 4 & 6 & 10 & 8 & 21 & 25 & 110 \\
\hline \multirow[t]{6}{*}{ Seedless } & $2001 / 2^{\circ}$ & $25 / 10 / 2001$ & 5 & 6 & 8 & 15 & 3 & 4 & 5 & 14 & 18 & 25 & 103 \\
\hline & $2002 / 1^{\circ}$ & $04 / 02 / 2002$ & 5 & 4 & 4 & 17 & 5 & 2 & 5 & 10 & 21 & 29 & 102 \\
\hline & $2002 / 1^{\circ}$ & $11 / 04 / 2002$ & 5 & 3 & 5 & 18 & 3 & 3 & 6 & 14 & 18 & 25 & 100 \\
\hline & \multicolumn{2}{|c|}{ Média } & 7 & 4 & 4 & 18 & 4 & 4 & 7 & 13 & 17 & 27 & 103 \\
\hline & \multicolumn{2}{|c|}{ Desvio padrão } & 3 & 2 & 2 & 3 & 1 & 2 & 2 & 3 & 5 & 4 & 6 \\
\hline & $2001 / 1^{\circ}$ & $19 / 12 / 2000$ & 4 & 2 & 3 & 16 & 3 & 6 & 5 & 16 & 13 & 25 & 93 \\
\hline & $2001 / 1^{\circ}$ & $24 / 04 / 2001$ & 11 & ---- & 3 & 12 & 4 & 8 & 5 & 12 & 9 & 29 & 94 \\
\hline \multirow[t]{6}{*}{ Seedless } & $2001 / 2^{\circ}$ & $20 / 06 / 2001$ & 6 & 5 & 3 & 22 & 4 & 6 & 10 & 8 & 21 & 25 & 110 \\
\hline & $2001 / 2^{\circ}$ & $26 / 10 / 2001$ & 5 & 4 & 6 & 8 & 3 & 6 & 6 & 9 & 18 & 31 & 96 \\
\hline & $2002 / 1^{\circ}$ & $05 / 02 / 2002$ & 6 & 4 & 4 & 14 & 5 & 2 & 5 & 13 & 11 & 37 & 101 \\
\hline & $2002 / 1^{\circ}$ & $12 / 04 / 2002$ & 5 & 3 & 6 & 16 & 3 & 2 & 5 & 9 & 22 & 35 & 106 \\
\hline & \multicolumn{2}{|c|}{ Média } & 6 & 4 & 4 & 15 & 4 & 5 & 6 & 11 & 16 & 30 & 100 \\
\hline & \multicolumn{2}{|c|}{ Desvio padrão } & 2 & 1 & 1 & 5 & 1 & 2 & 2 & 3 & 5 & 5 & 7 \\
\hline
\end{tabular}


TABELA 2 - Requerimentos térmicos (Graus-Dias) para os períodos compreendidos entre as principais fases fenológicas de cinco variedades de uvas sem sementes, Petrolina-PE, 2000 a 2002.

\begin{tabular}{|c|c|c|c|c|c|c|c|c|c|c|c|c|c|}
\hline \multirow[t]{2}{*}{ Variedades } & \multirow{2}{*}{$\begin{array}{c}\text { Ano/ } \\
\text { Semestre }\end{array}$} & \multirow[b]{2}{*}{$\begin{array}{c}\text { Data } \\
\text { de poda }\end{array}$} & \multicolumn{11}{|c|}{ Fases Fenológicas } \\
\hline & & & 1 & 2 & 3 & 4 & 5 & 6 & 7 & 8 & 9 & 10 & Total \\
\hline \multirow{8}{*}{$\begin{array}{l}\text { Superior } \\
\text { Seedless }\end{array}$} & $2001 / 1^{\circ}$ & $18 / 12 / 2000$ & 115,6 & 41,3 & 44,5 & 261,3 & 46,3 & 79,3 & 141,5 & 255,5 & 142,9 & 206 & 1334 \\
\hline & $2001 / 1^{\circ}$ & $25 / 04 / 2001$ & 189,0 & & 50,0 & 183,3 & 87,7 & 70,9 & 67,9 & 149,8 & 122,4 & 304,3 & 1225 \\
\hline & $2001 / 2^{\circ}$ & $21 / 06 / 2001$ & 98,2 & 45,4 & 36,8 & 243,5 & 46,5 & 71,1 & 99,2 & 97,5 & 216,3 & 133,5 & 1088 \\
\hline & $2001 / 2^{\circ}$ & $29 / 10 / 2001$ & 95,8 & 36,2 & 82,9 & 268,5 & 65,0 & 48,9 & 112,9 & 170,4 & 216,4 & 357,9 & 1455 \\
\hline & $2002 / 1^{\circ}$ & $29 / 01 / 2002$ & 84,2 & 54,9 & 70,6 & 211,9 & 61,4 & 87,5 & 75,0 & 172,8 & 232,5 & 438,3 & 1489 \\
\hline & $2002 / 1^{\circ}$ & $09 / 04 / 2002$ & 108,2 & 42,3 & 53,1 & 279,7 & 27,1 & 44,0 & 90,9 & 96,6 & 305,9 & 250,5 & 1298 \\
\hline & \multicolumn{2}{|c|}{ Média } & 115,2 & 44,0 & 56,3 & 241,4 & 55,7 & 67,0 & 97,9 & 157,1 & 206,1 & 281,8 & 1315 \\
\hline & \multicolumn{2}{|c|}{ Desvio Padrão } & 37,7 & 6,9 & 17,2 & 37,0 & 20,7 & 17,1 & 26,8 & 58,9 & 66,1 & 109,1 & 148 \\
\hline \multirow{8}{*}{ Perlette } & $2001 / 1^{\circ}$ & $19 / 12 / 2000$ & 102,2 & 41,3 & 44,5 & 261,3 & 62,9 & 77,9 & 158,2 & 222,5 & 175,3 & 555,1 & 1701 \\
\hline & $2001 / 1^{\circ}$ & $18 / 04 / 2001$ & 102,2 & & 55,5 & 263,2 & 46,0 & 101,5 & 57,6 & 119,2 & 234,2 & 337,3 & 1317 \\
\hline & $2001 / 2^{\circ}$ & $18 / 06 / 2001$ & 85,7 & 60,7 & 34,0 & 258,5 & 44,8 & 80,9 & 114,1 & 56,7 & 370,4 & 361,1 & 1467 \\
\hline & $2001 / 2^{\circ}$ & $23 / 10 / 2001$ & 93,4 & 47,9 & 102,5 & 221,2 & 64,5 & 79,2 & 66,0 & 160,5 & 403,6 & 291,3 & 1530 \\
\hline & $2002 / 1^{\circ}$ & $29 / 01 / 2002$ & 84,1 & 55,1 & 68,9 & 242,6 & 60,4 & 58,3 & 74,5 & 122,7 & 406,0 & 507,6 & 1680 \\
\hline & $2002 / 1^{\circ}$ & $08 / 04 / 2002$ & 110,3 & 56,2 & 53,1 & 249,0 & 57,7 & 28,1 & 106,8 & 108,9 & 326,7 & 293,6 & 1390 \\
\hline & \multicolumn{2}{|c|}{ Média } & 96,3 & 52,2 & 59,8 & 249,3 & 56,1 & 71,0 & 96,2 & 131,8 & 319,4 & 391,0 & 1514 \\
\hline & \multicolumn{2}{|c|}{ Desvio Padrão } & 10,3 & 7,6 & 24,0 & 15,9 & 8,6 & 25,1 & 37,8 & 55,6 & 95,1 & 112,9 & 155 \\
\hline \multirow{8}{*}{ Catalunha } & $2001 / 1^{\circ}$ & $18 / 12 / 2000$ & 115,6 & 41,3 & 44,5 & 261,3 & 46,3 & 94,6 & 174,5 & 269,8 & 179,5 & 463,3 & 1691 \\
\hline & $2001 / 1^{\circ}$ & $19 / 04 / 2001$ & 130,7 & & 70,0 & 265,3 & 43,2 & 102,7 & 42,6 & 119,2 & 234,2 & 304,3 & 1312 \\
\hline & $2001 / 2^{\circ}$ & $19 / 06 / 2001$ & 84,9 & 49,6 & 34,0 & 280,3 & 34,7 & 82,8 & 110,3 & 99,3 & 292,1 & 309,4 & 1377 \\
\hline & $2001 / 2^{\circ}$ & $24 / 10 / 2001$ & 91,7 & 48,2 & 103,4 & 48,9 & 79,3 & 49,0 & 81,3 & 268,0 & 236,3 & 302,9 & 1309 \\
\hline & $2002 / 1^{\circ}$ & $31 / 01 / 2002$ & 111,2 & 55,4 & 56,2 & 275,8 & 75,3 & 44,9 & 75,8 & 127,5 & 310,3 & 316,5 & 1449 \\
\hline & $2002 / 1^{\circ}$ & $10 / 04 / 2002$ & 92,1 & 42,3 & 53,1 & 262,3 & 41,6 & 59,2 & 87,9 & 166,3 & 235,3 & 289,2 & 1329 \\
\hline & \multicolumn{2}{|c|}{ Média } & 104,4 & 47,4 & 60,2 & 232,3 & 53,4 & 72,2 & 95,4 & 175,0 & 248,0 & 330,9 & 1411 \\
\hline & \multicolumn{2}{|c|}{ Desvio Padrão } & 17,6 & 5,8 & 24,3 & 90,2 & 18,9 & 24,5 & 44,5 & 75,9 & 46,9 & 65,5 & 147 \\
\hline \multirow{8}{*}{$\begin{array}{c}\text { Thompson } \\
\text { Seedless }\end{array}$} & $2001 / 1^{\circ}$ & $18 / 12 / 2000$ & 101,7 & 27,8 & 42,2 & 291 & 46,3 & 94,6 & 158,2 & 269,8 & 128 & 514,9 & 1675 \\
\hline & $2001 / 1^{\circ}$ & $23 / 04 / 2001$ & 189,4 & & 30,5 & 219,6 & 86,0 & 45,8 & 68,1 & 151,0 & 168,3 & 270,3 & 1229 \\
\hline & $2001 / 2^{o}$ & $20 / 06 / 2001$ & 84,2 & 60,1 & 35,6 & 268,2 & 46,5 & 71,1 & 110,3 & 99,3 & 292,1 & 375,5 & 1443 \\
\hline & $2001 / 2^{\circ}$ & $25 / 10 / 2001$ & 90,4 & 64,3 & 86,9 & 269,3 & 47,3 & 65,0 & 81,3 & 233,9 & 270,3 & 331,2 & 1540 \\
\hline & $2002 / 1^{\circ}$ & $04 / 02 / 2002$ & 81,0 & 58,0 & 57,5 & 274,9 & 75,1 & 30,3 & 75,8 & 159,6 & 320,5 & 287,8 & 1421 \\
\hline & $2002 / 1^{o}$ & $11 / 04 / 2002$ & 90,2 & 56,4 & 65,5 & 252,8 & 41,8 & 44,5 & 87,9 & 178,0 & 223,5 & 302,9 & 1343 \\
\hline & \multicolumn{2}{|c|}{ Média } & 106,2 & 53,3 & 53,0 & 262,6 & 57,2 & 58,6 & 96,9 & 181,9 & 233,8 & 347,1 & 1442 \\
\hline & \multicolumn{2}{|c|}{ Desvio Padrão } & 41,4 & 14,6 & 21,2 & 24,4 & 18,5 & 23,1 & 33,3 & 61,2 & 74,6 & 90,1 & 154 \\
\hline \multirow{8}{*}{ Seedless } & $2001 / 1^{\circ}$ & $19 / 12 / 2000$ & 74,1 & 28,2 & 41,3 & 234,0 & 43,0 & 92,8 & 77,9 & 254,5 & 204,9 & 390,8 & 1442 \\
\hline & $2001 / 1^{\circ}$ & $24 / 04 / 2001$ & 174,7 & & 47,6 & 49,4 & 56,3 & 102,3 & 68,1 & 151,0 & 109,2 & 350,7 & 1109 \\
\hline & $2001 / 2^{\circ}$ & $20 / 06 / 2001$ & 84,2 & 60,1 & 35,6 & 222,1 & 35,0 & 69,3 & 137,3 & 80,2 & 254,7 & 510,5 & 1489 \\
\hline & $2001 / 2^{\circ}$ & $26 / 10 / 2001$ & 91,9 & 65,7 & 101,3 & 139,4 & 49,4 & 79,8 & 97,9 & 144,5 & 304,4 & 404,4 & 1478 \\
\hline & $2002 / 1^{o}$ & $05 / 02 / 2002$ & 67,3 & 56,2 & 61,0 & 213,6 & 75,3 & 29,6 & 60,9 & 205,3 & 171,0 & 522,5 & 1463 \\
\hline & $2002 / 1^{\circ}$ & $12 / 04 / 2002$ & 88,3 & 27,9 & 78,6 & 226,4 & 55,2 & 31,1 & 75,7 & 108,9 & 281,7 & 429,2 & 1403 \\
\hline & \multicolumn{2}{|c|}{ Média } & 96,8 & 47,6 & 60,9 & 180,8 & 52,4 & 67,5 & 86,3 & 157,4 & 221,0 & 434,7 & 1397 \\
\hline & \multicolumn{2}{|c|}{ Desvio Padrão } & 39,3 & 18,2 & 25,1 & 73,0 & 13,8 & 30,9 & 27,9 & 63,6 & 73,5 & 68,4 & 144 \\
\hline
\end{tabular}

A 'Superior Seedless' foi a variedade mais precoce, cujo ciclo fenológico variou de 85 (podas em junho e outubro de 2001) a 100 dias (poda em janeiro de 2002), com uma média de 91 dias entre o estádio de gemas dormentes à plena maturação. Os requerimentos térmicos nesta variedade, variaram entre 1.088 e 1.489 GD (podas de junho de 2001 e janeiro de 2002, respectivamente). 'Marroo Seedless' foi a segunda variedade mais precoce, cujo ciclo fenológico variou entre 93 (poda em dezembro de 2000) e 110 dias (poda em junho de 2001), o que correspondeu à duração média de 100 dias desde a poda até a colheita e requerimentos térmicos que variaram entre 1.109 e 1.489 GD. A duração do ciclo fenológico variou de 88 a 113 dias na 'Perlette', 96 a 110 dias na 'Catalunha' e de 93 a 110 dias na 'Thompson Seedless'. Estas três variedades apresentaram comportamentos semelhantes quanto à duração do ciclo e requerimentos térmicos, respectivamente de 105, 102 e 103 dias e 1.514, 1.411 e $1.441 \mathrm{GD}$, para as médias das cinco épocas de poda. Esta semelhança é mais evidenciada entre a 'Catalunha' e a 'Thompson Seedless', onde a maior diferença na duração do ciclo entre as variedades foi de apenas 6 dias para a poda de outubro de 2002. A variedade Perlette destacou-se entre todas as variedades estudadas por ser a mais tardia e que demanda maior somatório térmico para completar o ciclo. A duração média do ciclo para as variedades Superior Seedless, Marroo Seedless e Thompson Seedless são semelhantes àquelas obtidas em trabalhos anteriores para estas variedades nesta mesma região (Leão e Pereira, 2001; Grangeiro et al., 2002). Entretanto, pode-se observar uma redução de 86 dias no ciclo da variedade Thompson Seedless quando comparada aos resultados obtidos por Villaseca et al. (1986) no Chile. Os requerimentos térmicos desde a poda até a colheita, observados neste trabalho, estão próximos dos valores obtidos por Pedro Júnior et al. (1993) na variedade Niágara rosada em diferentes regiões do Estado de São Paulo e Mandelli (1984) em Bento Gonçalves para o período brotaçãocolheita.

O estádio fenológico de amadurecimento de frutos compreendido entre o início e o final da maturação foi o que apresentou maior duração em todas as cultivares, bem como maior demanda térmica. Nas cultivares 'Superior Seedless' e 'Marroo Seedless', a poda que promoveu a maior duração desta fase do ciclo foi aquela realizada no final de janeiro e início de fevereiro de 2002, quando obteve-se respectivamente 31 e 37 dias nas duas variedades. Por outro lado, para as demais varieda- 
des, a poda realizada em dezembro de 2000 foi a que proporcionou maior duração deste estádio fenológico, sendo de 37, 33 e 31 dias para Perlette, Thompson Seedless e Catalunha, respectivamente. Outros estádios fenológicos que também apresentaram duração mais prolongada que os demais foram a fase final de crescimento de bagas (1/2 baga à início de maturação) cuja média variou de 15 dias na 'Superior Seedless' a 21 dias na 'Perlette', e o período compreendido entre 4 a 6 folhas separadas e início de floração (15 dias na 'Marroo Seedless', 17 dias na 'Superior Seedless' e 'Perlette' e 18 dias na 'Thompson Seedless' e 'Catalunha'). As fases fenológicas desde $1 / 2$ baga até o final de maturação foram as que exigiram maior somatório térmico em todas as variedades (Tabela 2).

\section{CONCLUSÕES}

Nas condições em que foi realizado este trabalho, pode-se concluir o seguinte:

1) As variedades mais precoces foram a 'Superior Seedless' com duração média do ciclo fenológico de 91 dias e Marroo Seedless com 100 dias

2) 'Perlette', 'Thompson Seedless' e 'Catalunha' apresentam comportamento fenológico semelhante com média de duração para o ciclo fenológico de 105, 103 e 102 dias, respectivamente;

3) Os requerimentos térmicos para completar o ciclo fenológico foram mais elevados nas variedades Perlette (1514 GD), Thompson Seedless (1442 GD) e Catalunha (1411 GD), enquanto 'Superior Seedless' (1315 GD) e 'Maroo Seedless' (1397 GD) foram menos exigentes;

4) Os períodos compreendidos entre o início e o final da maturação, 1/2 baga a início de maturação e 5 a 6 folhas separadas a início de floração apresentaram a maior duração entre todos os períodos em todas as variedades.

\section{REFERÊNCIASBIBLIOGRÁFICAS}

BOLIANI, A.C. Avaliação fenológica de videiras Vitis vinifera L. cvs. Itália e Rubi na região oeste do estado de São Paulo. 1994. 188f. Tese (Doutorado em Produção Vegetal) - Faculdade de Ciências Agrárias e Veterinárias, Universidade Estadual Paulista, Jaboticabal, 1994.

FERRI, C. P. Caracterização agronômica e fenológica de cultivares e clones de videira (Vitis spp) mantidos no Instituto Agronômico, Campinas, SP. 1994. 89f. Dissertação (Mestrado em Fitotecnia) Escola Superior de Agricultura "Luiz de Queiroz", Universidade de São Paulo, Piracicaba, 1994

GRANGEIRO, L. C.; LEÃO, P C. de S.; SOARES, J. M. Caracterização fenológica e produtiva da variedade de uva Superior Seedless cultivada no Vale do São Francisco. Revista Brasileira de Fruticultura, Jaboticabal, SP, v.24, n.2, p.552-554, 2002.

GUERREIRO, V. M. Avaliação fenológica da videira (Vitis labrusca L. $\mathbf{x}$ Vitis vinifera L.) cultivar Niagara Rosada na região de Selvíria MS. 1997. 98f. Dissertação (Mestrado em Sistema de Produção) Faculdade de Engenharia, Universidade Estadual Paulista, Ilha Solteira, 1997.

LEÃO, P. C. de S.; PEREIRA, F. M. Estudo da brotação e da fertilidade das gemas de cultivares de uvas sem sementes nas condições tropicais do Vale do Submédio São Francisco, Revista Brasileira de Fruticultura, Jaboticabal, v.23, n.1, p.30-34, 2001.

LORENZ, D. H.; EICHHORN, K. W.; BLEIHOLDER, H.; KLOSE, R.; MEIER, U.; WEBER, E. Phenological growth stages of the grapevine (Vitis vinifera L. ssp. vinifera) - Codes and descriptions according to the extended BBCH scale. Australian Journal of Grape and Wine Research, v.1, p.100-3, 1995.

MANDELLI, F. Comportamento fenológico das principais cultivares de Vitis vinifera L. para a região de Bento Gonçalves, RS. 1984. 125f. Dissertação (Mestrado em Agrometeorologia) - Escola Superior de Agricultura “Luiz de Queiroz", Universidade de São Paulo, Piracicaba, 1984.

PEDRO JÚNIOR, M. J.; SENTELHAS, P. C.; MARTINS, F. P. Previsão agrometeorológica da data de colheita para a videira 'Niágara Rosada'. Bragantia, Campinas, v.53, n.1, p.113-9, 1994.

PEDRO JÚNIOR, M. J.; SENTELHAS, P. C.; POMMER, C. V.; MARTINS, F. P.; GALLO, P. B.; SANTOS, R. R. dos; BOVI, V.; SABINO, J. C. Caracterização fenológica da videira 'Niagara Rosada' em diferentes regiões paulistas. Bragantia, Campinas, v.52, n.2, p.153-160, 1993.

SILVA, A. C.; PEREIRA, F. M.; MARTINS, F. P. Comportamento de cultivares americanas de videira na região de Jundiaí - SP. Científica, Jaboticabal, v.18, n.1, p.61-70, 1990.

TERRA, M. M. et al. Tecnologia para produção de uva Itália na região noroeste do estado de São Paulo. 2. ed. Campinas: CATI, 1998. 58p. (Documento Técnico, 97).

VILLA NOVA, N.A.; PEDRO JÚNIOR, M. J.; PEREIRA, A. R.; OMETTO, J. C. Estimativas de grus-dias acumulados acima de qualquer temperatura-base, em função das temperaturas máxima e mínima. São Paulo: Universidade de São Paulo- Instituto de Geografia, 1972. 8p. (Caderno de Ciências da Terra, 30).

VILLASECA, S.; NOVOA, R.; MUÑOZ, I. Fenologia y sumas de temperaturas en 24 variedades de vid. Agricultura Tecnica, Santiago, v.46, n.1, p.63-7, 1986. 